\title{
Small Antenna Based on MEMS and Metamaterial Properties for Reconfigurable Applications
}

\author{
G. Rosas-Guevara, ${ }^{1}$ R. Murphy-Arteaga, ${ }^{1}$ and W. Moreno ${ }^{2}$ \\ ${ }^{1}$ Department of Electronics, Instituto Nacional de Astrofísica, Óptica y Electrónica (INAOE), 72840 Puebla, PUE, Mexico \\ ${ }^{2}$ Department of Electrical Engineering, University of South Florida, Tampa, FL 33620, USA \\ Correspondence should be addressed to R. Murphy-Arteaga; rmurphy@inaoep.mx
}

Received 22 June 2012; Revised 4 December 2012; Accepted 7 December 2012

Academic Editor: Francisco Falcone

Copyright ( 2013 G. Rosas-Guevara et al. This is an open access article distributed under the Creative Commons Attribution License, which permits unrestricted use, distribution, and reproduction in any medium, provided the original work is properly cited.

\begin{abstract}
This paper presents the design of a novel, small coplanar antenna using microelectromechanical systems (MEMS) and metamaterial (MTM) properties. The antenna is designed using coplanar waveguide (CPW) technology, presenting lower dielectric losses and higher signal integrity. The design method for this MEMS-MTM antenna, herein presented, is based on a composite right/left hand (CRLH) transmission Line (TL) using a mixed approach; considering the circuit model and full-wave simulations. The fabrication process is based on high-resistivity silicon wafers. The radiator has dimensions of $0.017 \lambda_{g} \times 0.033 \lambda_{g}$ and a thickness of $0.0116 \lambda_{g}$, whereas the complete circuit, of $5 \mathrm{~mm} \times 11 \mathrm{~mm}$, is equivalent to $0.14 \lambda_{g} \times 0.31 \lambda_{g}$. The antenna is designed using MEMS parallelplate capacitors as the radiator, which also allows for the reconfiguration of the central frequency by electrostatically varying the capacitance. The results presented here correspond to a central frequency of $8.4 \mathrm{GHz}$. Due to its small size, this antenna has a wide variety of applications in wireless circuits for different fields.
\end{abstract}

\section{Introduction}

The advancement of technology is due, in large part, to the judicious incorporation of new techniques as they become available and mature. Electronic devices are a clear example of this; they have evolved from very simple structures to complete systems being fabricated on chip. Nowadays, two emerging technologies with a wide application in electronics are metamaterials [1-5] and microelectromechanical systems (MEMS). Using these in conjunction allows us to design and fabricate a plethora of new devices, especially in the field of high-frequency electronics, which is increasingly in demand. For a host of wireless applications, miniaturization, reconfigurability, better performance, more functions, higher speeds, and higher output at lower costs are desired, as well as efficient and trustworthy connectivity. Hence, a fundamental component is an antenna, which has to be easily integrated with associated circuitry in an IC.

This paper presents the design of a novel antenna, based on MEMs and metamaterial properties, which can be used for many high-frequency applications. The objective of this work is twofold: innovating RF-MEMS-MTM circuits through an original design method and a novel fabrication process, and developing ad-hoc designs for RF-MEMS-MTM circuits using surface-micromachining technology, which is fully compatible with most integrated circuit fabrication processes. These circuits can then achieve high performance, low cost, and compact size. In this paper, we present the design, fabrication, and characterization of a novel MEMS-MTM antenna using a CRLH-TL (Composite Right-Left Hand Transmission Line) structure and two MEMS capacitors as a 3D element to tune the antenna in frequency. An electrode of these capacitors also functions as the radiating element, or antenna.

The paper is organized as follows: Section 2 first presents a brief description of the antenna, then establishes a metamaterial model for the antenna, and finally presents all the details of the MEMS capacitor as a 3D structure. Section 3 explains the fabrication process flow. Section 4 presents simulation and measurements results. Finally, in Section 5 the most relevant conclusions are discussed. 


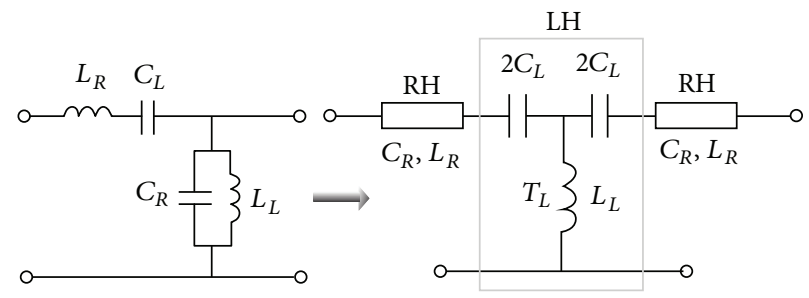

FIGURE 1: Electronic emulation of metamaterial properties and its equivalent circuit.

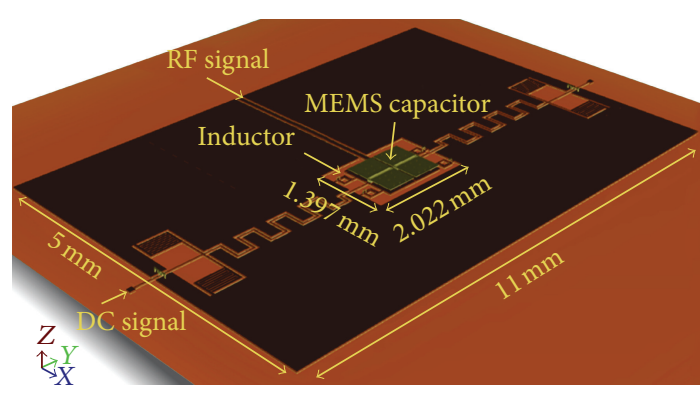

(a)

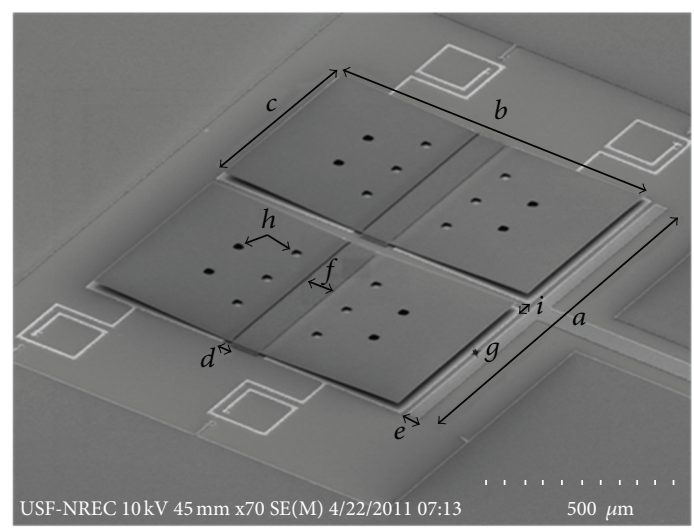

(b)

Figure 2: (a) MEMS-MTM antenna in CoventorWare and (b) details of a MEMS-MTM CRLH-TL basic cell of the fabricated antenna.

\section{MEMS-MTM Antenna Design}

The main idea behind this antenna is miniaturization. The smaller an antenna is, conserving all its important characteristics, the more applications it can cover. It is well known that, using metamaterial concepts, small sizes can be readily achieved. In an electronic circuit, metamaterial properties can be emulated with the use of shunt inductors and series capacitors together with the shunt capacitors and series inductors common in transmission line modeling, as shown in Figure 1. If these capacitors are variable, moreover, then the resonant frequency of the circuit can be changed, and the device becomes a tunable one. This can be easily
TABLE 1: Antenna dimensions.

\begin{tabular}{llc}
\hline Symbol & Description & Dimensions $[\mu \mathrm{m}]$ \\
\hline$a$ & Length of the coupled line & 1200 \\
$b$ & Length of the mobile electrode & 1000 \\
$c$ & Width of the mobile electrode & 576 \\
$d$ & $\begin{array}{l}\text { Distance between the anchor and } \\
\text { fixed electrode }\end{array}$ & 10 \\
$e$ & Width of the coupled line & 50 \\
$f$ & Width of the anchor & \\
$g$ & Gap between the coupled line & 5 \\
$h$ & and MEMS capacitors & $15 \times 15$ \\
$i$ & Holes & 30 \\
\hline
\end{tabular}

implemented using MEMS capacitors, whose capacitance can be changed by varying the gap between electrodes.

An antenna based on these criteria is the subject matter of this paper, and the most relevant steps in its design, fabrication, and characterization will be discussed in this section.

2.1. Description of the MEMS-MTM Antenna. The MEMSMTM antenna consists of a CRLH-MTM structure with two double MEMS capacitors and four inductors connected to ground, as shown in Figure 2(a). The MEMS capacitors are of the parallel-plate type. Additionally, a CPW RF choke is implemented as a feed line to control the MEMS capacitors. The RF choke is designed using a coplanar bias- $T$ line with a 90-degree equivalent length, loaded with $0.845 \mathrm{pF}$ due to the interdigitated capacitors, so that it approaches an open circuit at high frequencies, thus isolating the RF signals from the DC bias line and not having an impact on the microwave performance of the device.

The MEMS-MTM antenna feed is electromagnetically coupled with a CPW line, designed for a central frequency of $8.4 \mathrm{GHz}$ and a characteristic impedance of $50 \Omega$. The line spacing is $50 \mu \mathrm{m}$ and the signal line width is $78 \mu \mathrm{m}$. The dimensions of the antenna, including the DC bias line, are of $5 \times 11 \mathrm{~mm}\left(0.14 \lambda_{g} \times 0.31 \lambda_{g}\right)\left(\lambda_{g}\right.$ is the guided wavelength $)$. The basic cell, formed by the two double MEMS capacitors and four inductors, is $1.397 \times 2.022 \mathrm{~mm}\left(0.039 \lambda_{g} \times 0.057 \lambda_{g}\right)$ in size, as shown in Figures 2(a) and 2(b). The biasing scheme can be observed in these figures as an electromagnetically coupled transmission line with a $5 \mu \mathrm{m}$ gap $\left({ }^{*} g\right)$ between the MEMS capacitors and the transmission line. Table 1 displays the antenna dimensions, which are indicated in Figure 2(b). 


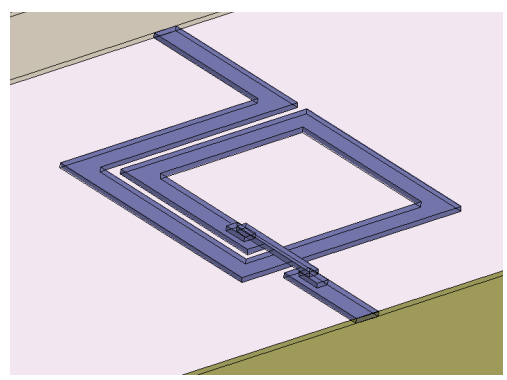

(a)

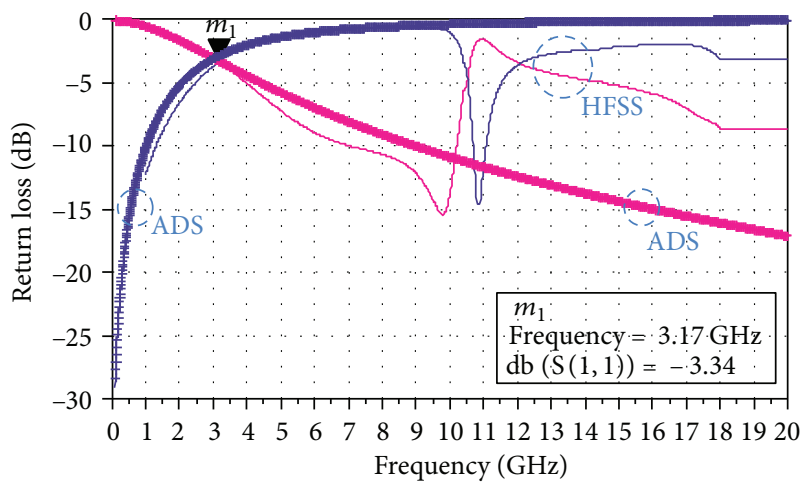

(c)

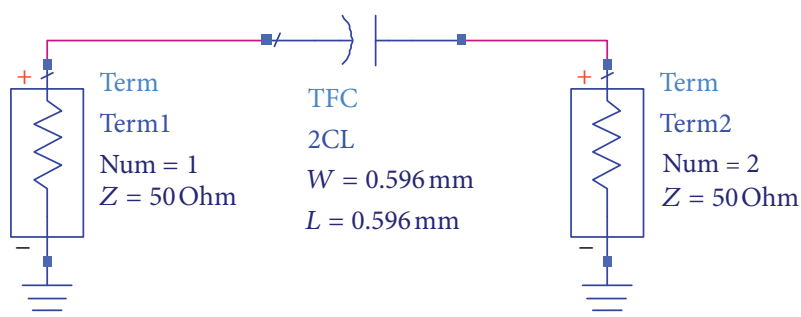

(e)

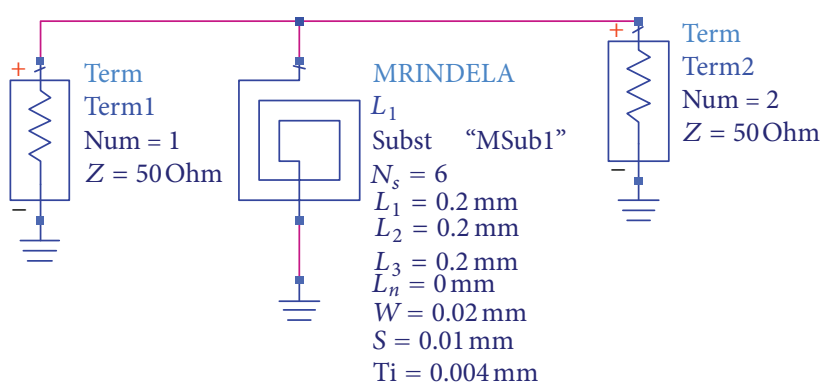

(b)

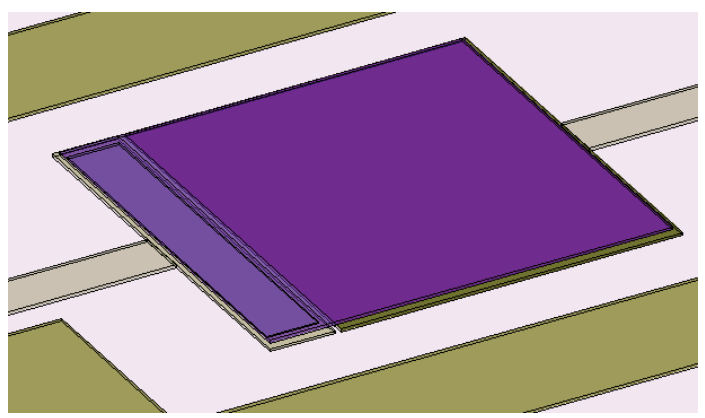

(d)

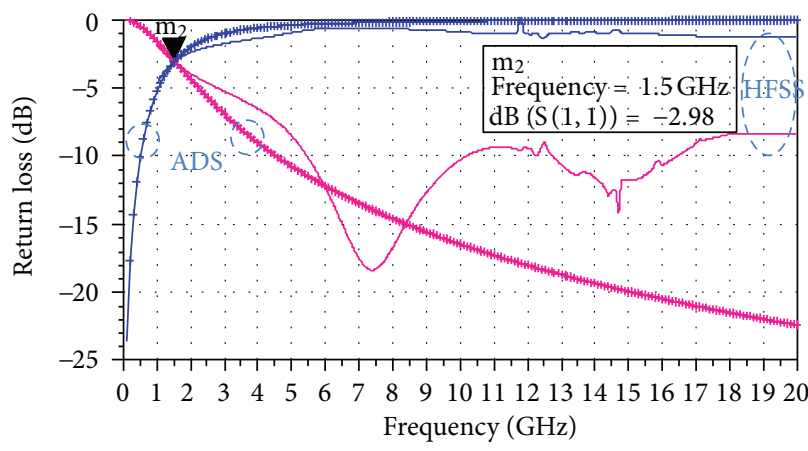

(f)

FIGURE 3: Performance of each antenna element according to ADS and HFSS: (a) full-wave simulation of the inductor in HFSS; (b) electric circuit model for the inductor in ADS; (c) simulated $\mathrm{S}_{11}$ inductor performance; (d) full-wave simulation of the capacitor in HFSS; (e) electric circuit model for the capacitor in ADS; (f) simulated $S_{11}$ capacitor performance.

2.2. MTM-Antenna Model. Metamaterials can be described using different models; this work focuses on a composite right/left handed (CRLH) transmission line (TL), which consists of dual elements, series $C$ and shunt $L$, loading a classical transmission line as displayed in Figure 1, where $L_{R}$, $C_{L}, C_{R}$, and $L_{L}$ are the constitutive elements.

The design methodology is based on a mixed approach using the developed circuit model based on a CRLH-TL structure and full-wave simulations.

Step 1. The parameters for a basic CRLH-MTM cell are obtained using (1) through (5), developed by Caloz and Itoh [3], once the correct modifications in sign and phase have been performed. The basic initial parameters are the central frequency $\left(\omega_{0}\right)$, coupling impedance $\left(Z_{C}\right)$, number of cells $(N)$, and RH/LH phases $\left(\varphi_{\mathrm{RH}}, \varphi_{\mathrm{LH}}\right)$, which are used to calculate the element values:

$$
\begin{gathered}
L_{L}=-\frac{2 N Z_{c}}{\omega_{0}\left[\varphi_{\mathrm{RH}}-\varphi_{\mathrm{LH}}\right]}, \\
L_{R}=-\frac{Z_{c}\left[\varphi_{\mathrm{RH}}-\varphi_{\mathrm{LH}}\right]}{2 N \omega_{0}}, \\
C_{R}=-\frac{\left[\varphi_{\mathrm{RH}}-\varphi_{\mathrm{LH}}\right]}{2 N Z_{c} \omega_{0}}, \\
C_{L}=-\frac{2 N}{\omega_{0} Z_{c}\left[\varphi_{\mathrm{RH}}-\varphi_{\mathrm{LH}}\right]}, \\
Z_{C}=Z_{L}=\sqrt{\frac{L_{L}}{C_{L}}}=Z_{R}=\sqrt{\frac{L_{R}}{C_{R}} .}
\end{gathered}
$$




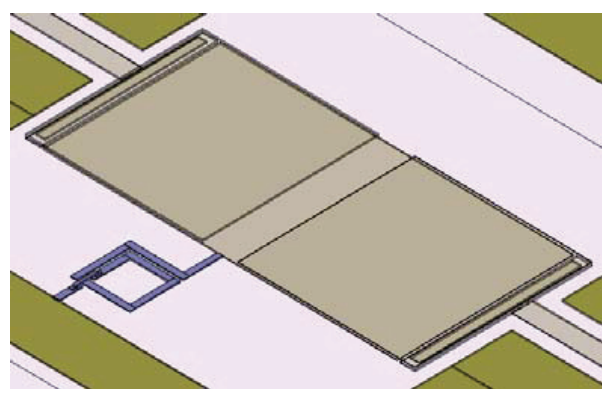

(a)

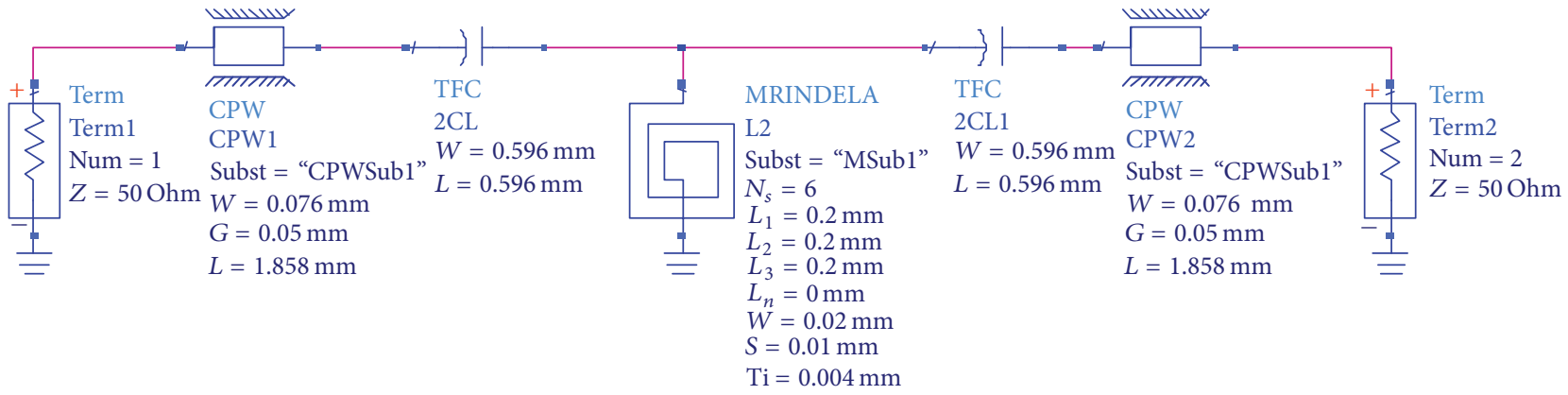

(b)

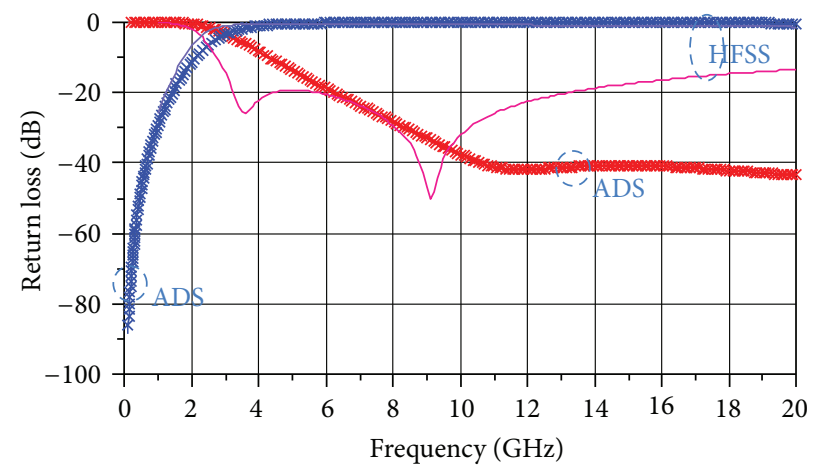

K

(c)

FIGURE 4: Performance of the CRLH-TL structure according to ADS and HFSS: (a) full-wave simulation of the CRLH-TL in HFSS; (b) electric circuit model for the inductor in ADS; (c) simulated $\mathrm{S}_{11}$ inductor performance.

The structure shown in Figure 1 was then simulated using advanced design system (ADS) with the values calculated from (1) to (5).

Step 2. Circuit implementation is determined. In this case $C=2 C_{L}$, and each element of this structure is independently simulated using ADS and Ansoft HFSS $[6,7]$, as shown in Figures 3(a), 3(b), 3(c), 3(d), 3(e), and 3(f). This is done in order to verify that there are no resonant frequencies in the operating range of the antenna.

Step 3. The complete CRLH-TL structure is simulated with distributed models and optimized with respect to these parameters using ADS and Ansoft HFSS, as shown in Figures 4(a), 4(b), and 4(c).
Step 4. Finally, some iterations in the simulation of the transmission line are made in order to update the loading elements to match them to the required equivalent circuit values, as obtained in Step 3.

The equivalent circuit model of the antenna is represented by the left hand part of the CRLH-TL structure. Figure 5(a) shows an MEMS-MTM antenna cell, and Figure 5(b) presents the response when the gap of the mobile electrode is changed from 4 to 30 micrometers.

2.3. MEMS Capacitor. The MEMS capacitors were designed as two back-to-back cantilevered structures, supported by an anchor in between, as shown in Figure 6. The gap between the plates is varied electrostatically, from a minimum of 4 microns to a maximum of 30 microns. For this work, 


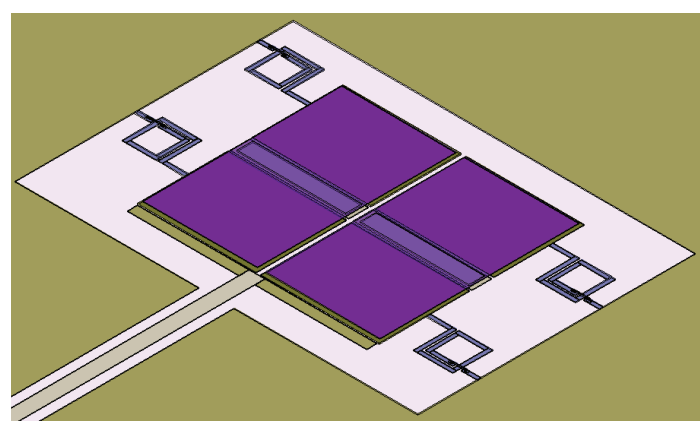

(a)

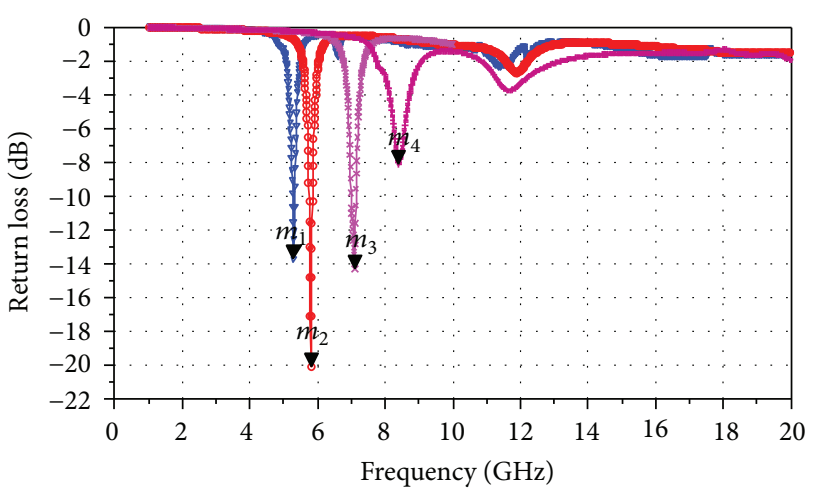

dB (gap_4_micrometers. ..S $(1,1)$ )

dB (gap_5_micrometers. ..S $(1,1)$ )

$\rightarrow \mathrm{dB}$ (gap_10_micrometers. . .S $(1,1))$

$\ldots$ dB (gap_30_micrometers...S $(1,1)$ )

(b)

FIgURE 5: Full-wave simulation for the antenna using HFSS: (a) sketch of the antenna, and (b) simulated $S_{11}$ parameter, where $m_{1}=$ $5.3 \mathrm{GHz}$ at $-13.747 \mathrm{~dB} ; m_{2}=5.8 \mathrm{GHz}$ at $-20.156 \mathrm{~dB} ; m_{3}=7.1 \mathrm{GHz}$ at $-14.323 \mathrm{~dB}$; and $m_{4}=8.4 \mathrm{GHz}$ at $-8.083 \mathrm{~dB}$.

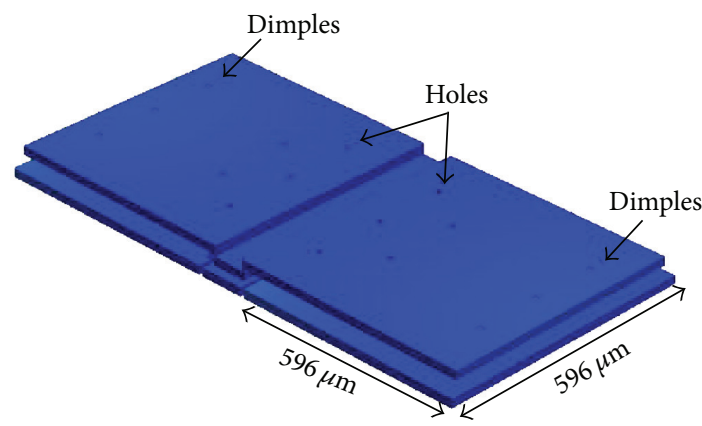

Figure 6: Details of the MEMS capacitor.

each capacitor was designed with an area of $596 \mu \mathrm{m} \times$ $596 \mu \mathrm{m}$, presenting a nominal capacitance value of $1.048 \mathrm{pF}$, which corresponds to $2 C_{L}$ in the equivalent circuit shown in Figure 1.

A detailed electrical-mechanical analysis was performed using CoventorWare [8], which was then used to determine

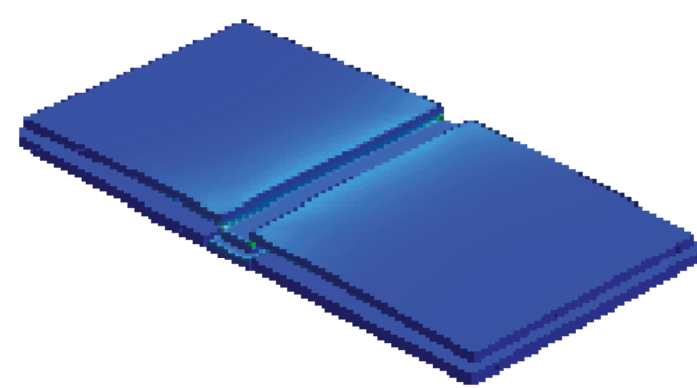

(a)

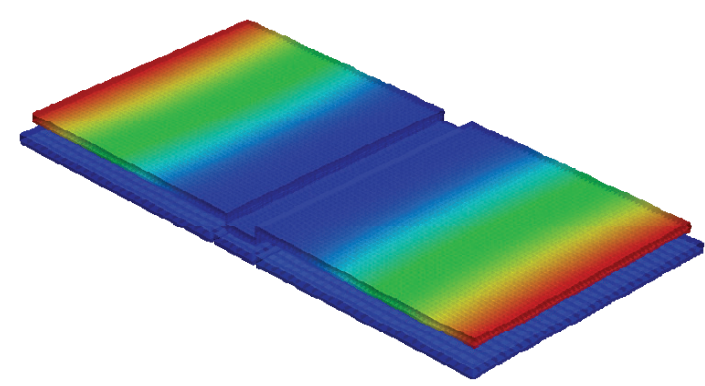

(b)

FIGURE 7: (a) Anchor stress simulations and (b) MEMS capacitor stress simulations along the $y$ axis.

the critical parameters such as the pull-in voltage, defined in [9], and the effective stiffness [10], presented in [11]. A rendering of the results is shown in Figures $7(\mathrm{a})$ and 7(b). Figure 7 (a) shows a mechanical simulation indicating the stress presented on the anchor of the MEMS capacitor, and the different shades in Figure 7(b) indicate the distribution of mechanical stresses along the length of the mobile electrode.

The deformations at the end of the electrodes and the stress on the anchor caused by electrode size and the movement of the electrodes were considered in the design. The results indicated that the optimal size for the anchor was $22 \%$ of electrode size, which allows for large displacements between them while avoiding collapse.

\section{Fabrication Process}

The fabrication process is based on surface micromachining technology, which under the implemented process conditions constitutes a novel fabrication technique, in the sense that it can naturally evolve for reconfigurable MEMS devices.

Figure 8 illustrates the fabrication process of the MEMSMTM antenna. It consists of five materials and four levels of masks on a high-resistivity silicon wafer (N type, $\langle 100\rangle$ orientation, $4000-8000 \Omega \cdot \mathrm{cm}$ resistivity and $490-530 \mu \mathrm{m}$ thickness). Firstly, silicon dioxide is grown on the silicon wafer as an electrical and thermal insulator, as displayed in Figure 8(a). Each MEMS capacitor of the antenna consists of two metal layers (bilayer), formed by titanium (Ti) and aluminum (Al), which are deposited as structural materials by sputtering, with one suspended level for the mechanical structures. Each metal layer consists of a thickness of $0.5 \mu \mathrm{m}$ of titanium and $2 \mu \mathrm{m}$ of aluminum, to avoid losses due 


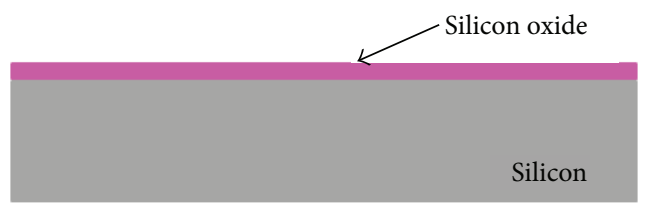

(a)

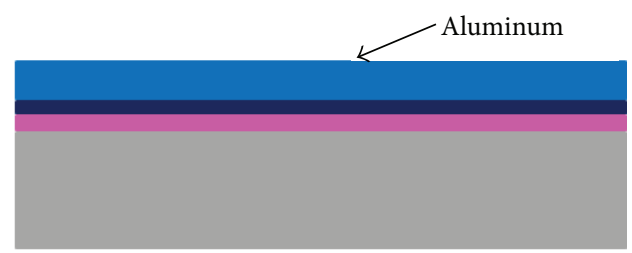

(c)

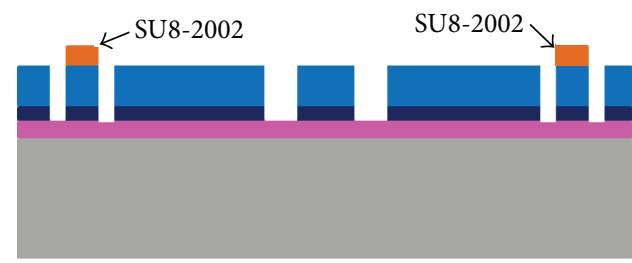

(e)

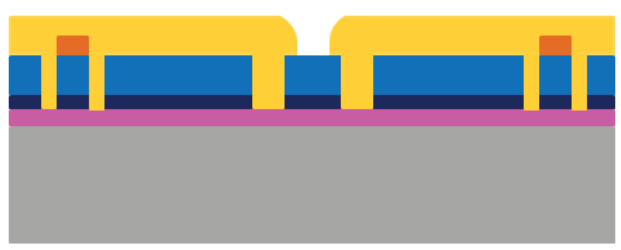

(g)

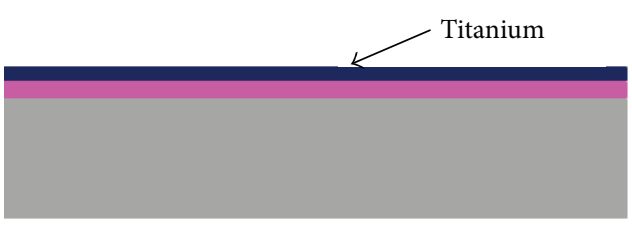

(b)

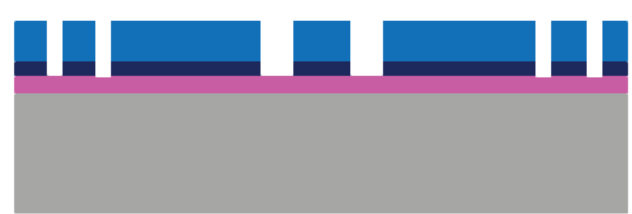

(d)

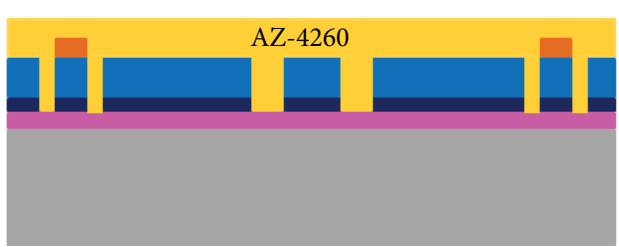

(f)

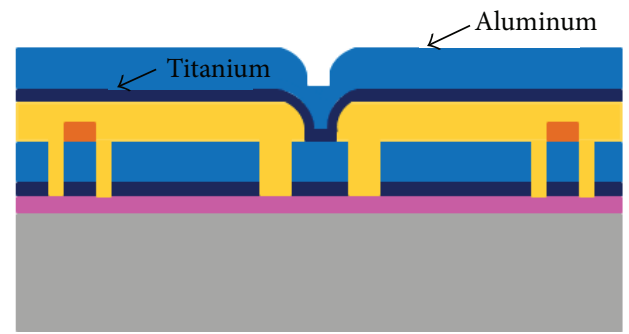

(h)

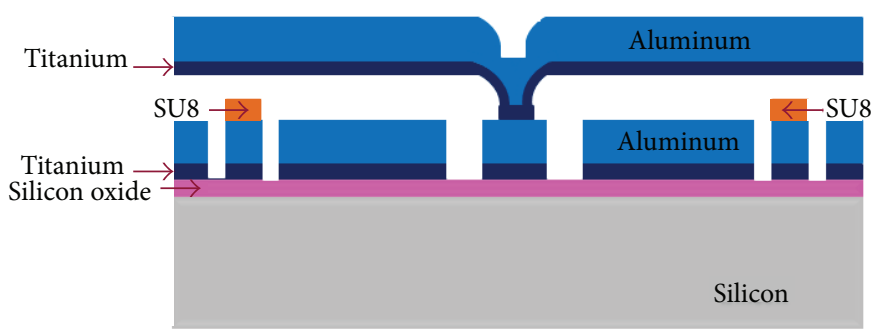

(i)

Figure 8: Fabrication process. (a) Standard wafer cleaning followed by the thermal growth of $\mathrm{SiO}_{2}$, (b) $0.5 \mu$ m-thick titanium deposited by sputtering, (c) $1.5 \mu \mathrm{m}$-thick aluminum deposited by sputtering, (d) ground plane patterned with Ti and Al by lithography, (e) dimples formed by SU8-2002 pattering, (f) AZ-4260 sacrificial layer forming, (g) definition of anchor by pattering, (h) Ti-Al deposited by sputtering and (i) MEMS release process.

to skin depth and to provide the structure a more robust mechanical stability, as shown in Figures 8(b), 8(c), and 8(d). Subsequently, SU8-2002 was deposited on the first metal bilayer as a dielectric, and the dimples were formed, as shown in Figure 8(e). A $5 \mu \mathrm{m}$-thick sacrificial layer of AZ-4620 was then deposited, and the structure was spin coated with positive photoresist $(\mathrm{PR}+)$ and patterned by UV lithography, as can be seen Figures 8(f) and 8(g). The patterned sacrificial layer was then thermally cured. Then, a second $2 \mu \mathrm{m}$-thick metal layer was deposited by sputtering. In this step, the mobile electrodes and bridge structures were formed, as displayed in Figure 8(h). Finally, the structures were released, as illustrated in Figure 8(i).

Figure 9 shows scanning electron microscope (SEM) photographs of the MEMS capacitor, MEMS-MTM antenna and a ZOR resonator. 


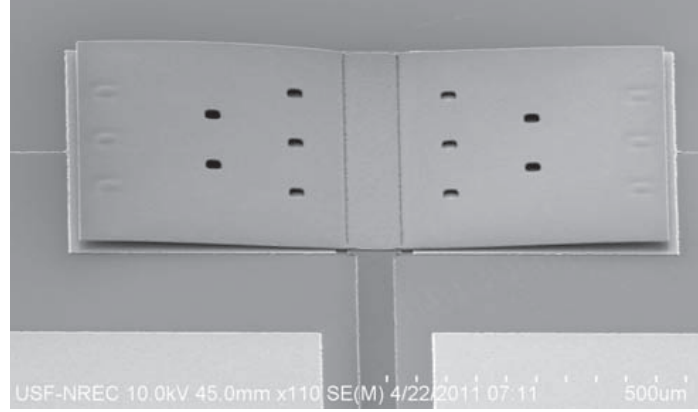

(a)

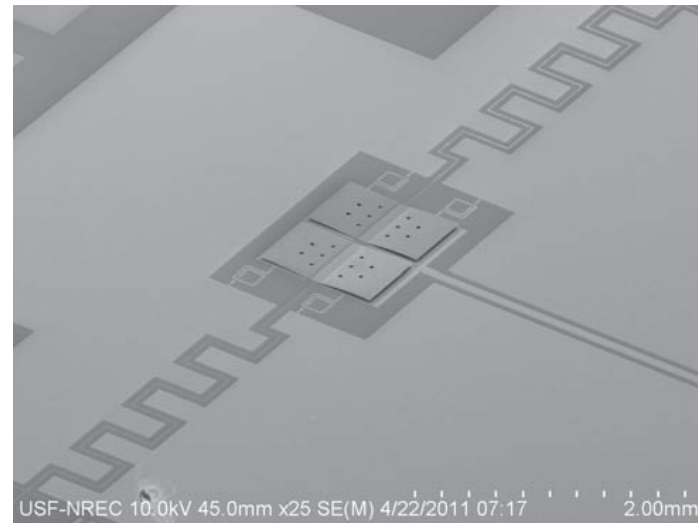

(b)

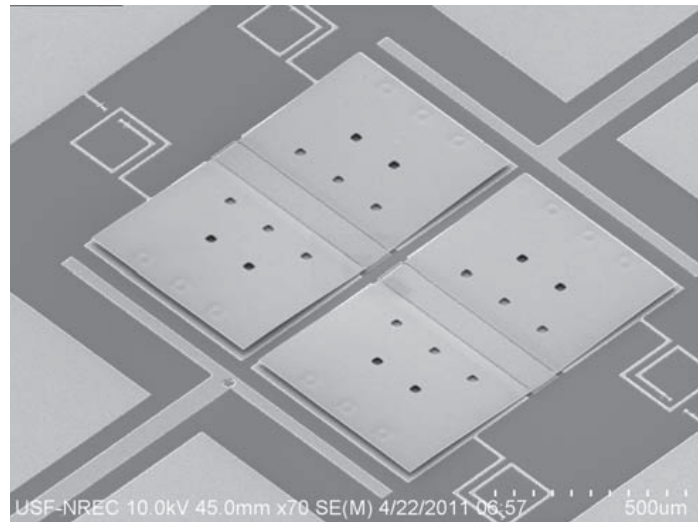

(c)

FIGURE 9: SEM photographs of (a) the MEMS capacitor, (b) a MEMS-MTM antenna basic cell, and (c) a ZOR resonator.

\section{Simulations and Measurements}

The simulations and results presented in this section are at a frequency of $8.4 \mathrm{GHz}$, which corresponds to the fabricated $30 \mu \mathrm{m}$ gap between electrodes in the MEMS capacitors.

Full-wave simulations using HFSS were performed and are shown in Figure 10, which is a $3 \mathrm{D}$ rendering of the radiation pattern and the gain.

Figures 11(a) and 11(b) illustrate the far-field radiation pattern simulated in the $E$ phi-plane $\left(x-z\right.$ plane, $\left.\mathrm{Phi}=0^{\circ}\right)$ and $H$ theta-plane $\left(y-z, \mathrm{Phi}=90^{\circ}\right)$ at $8.65 \mathrm{GHz}$ when the antenna presents a $30 \mu \mathrm{m}$ gap between electrodes.
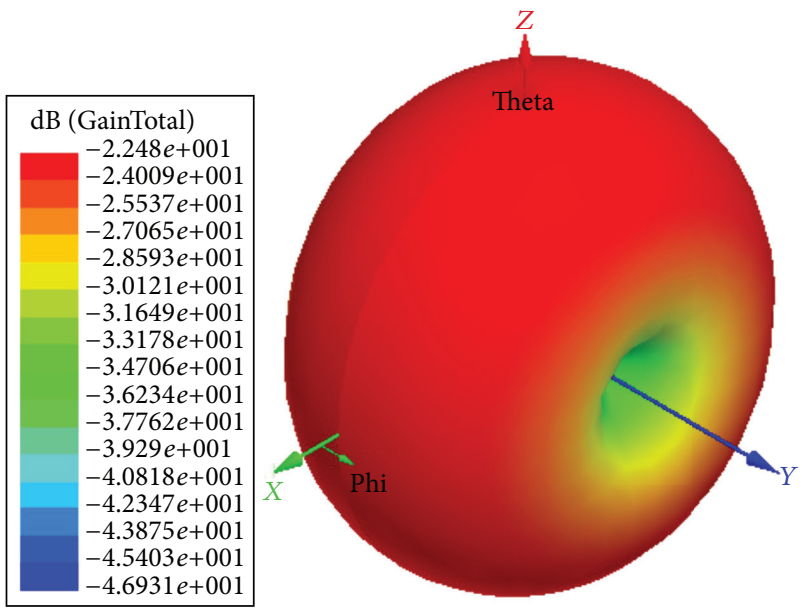

(a)
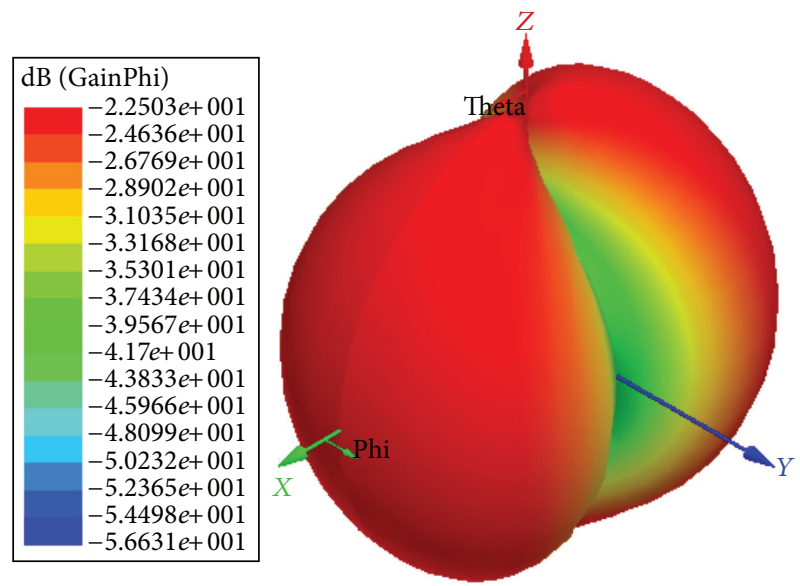

(b)

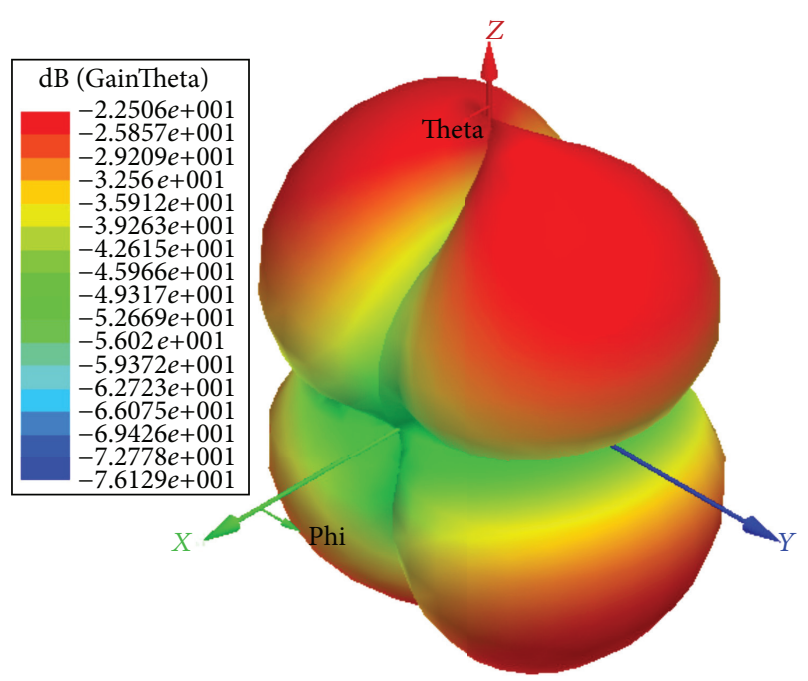

(c)

FIGURE 10: Simulated far-field radiation patterns at $8.4 \mathrm{GHz}$. (a) Total gain, (b) electric-plane gain and (c) magnetic-plane gain for the MEMS-MTM antenna. 


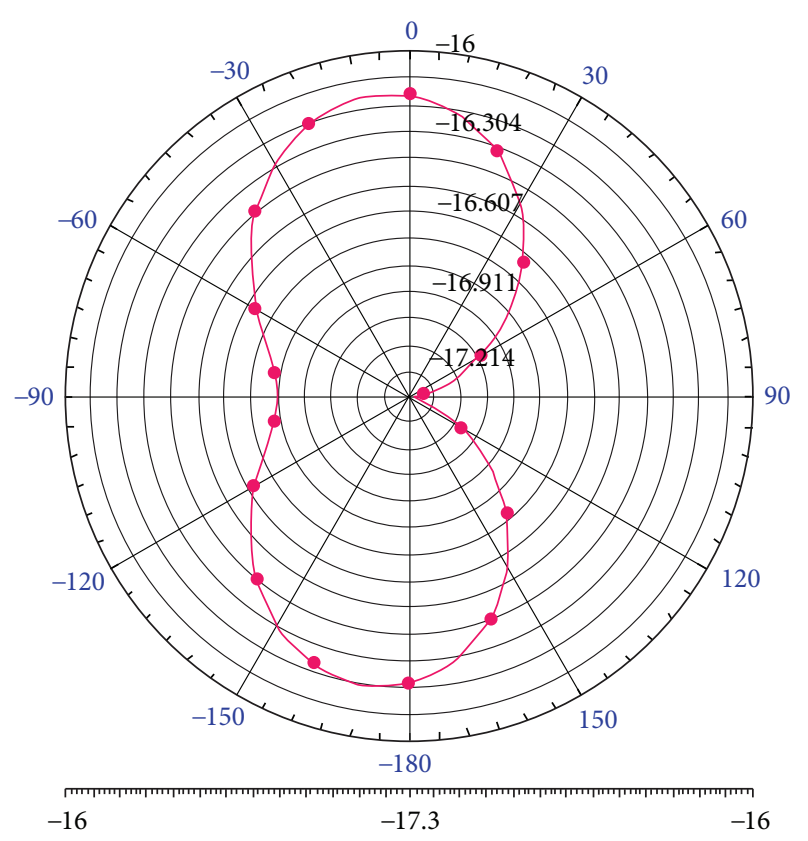

(a)

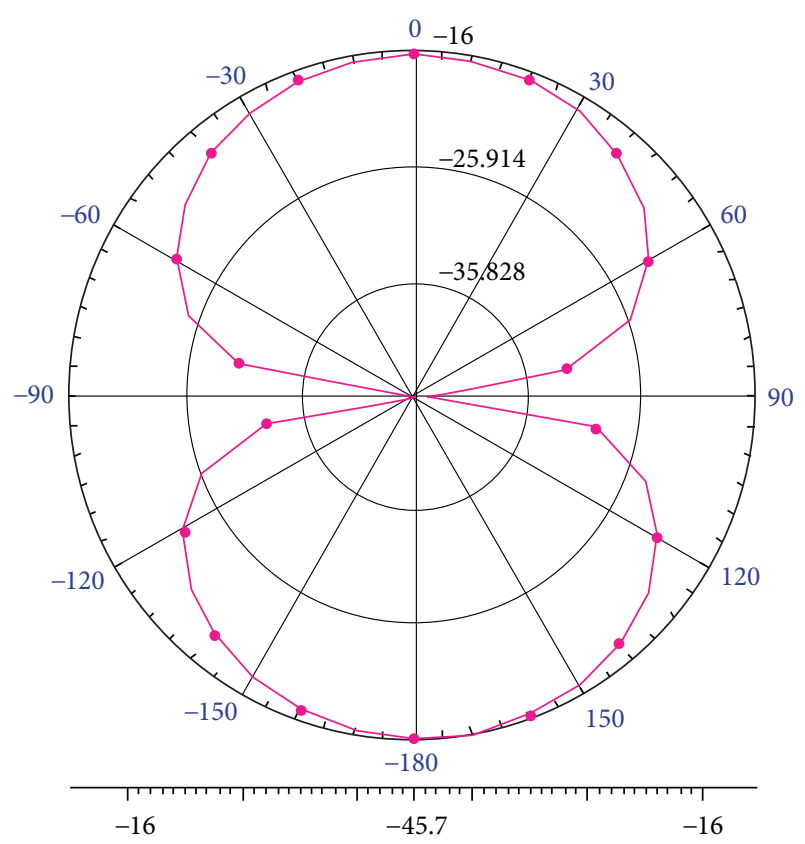

(b)

FIGURE 11: Simulated far-field radiation patterns (a) E-plane and (b) $H$-plane of the MEMS-MTM antenna at a frequency of $8.65 \mathrm{GHz}$.

The antenna measurements were performed on wafer using a previously calibrated vector network analyzer and a probe station.

Figure 12 shows the $S_{11}$ dispersion parameters versus frequency of the MEMS-MTM antenna. In this case, the antenna displayed a resonant frequency at $8.65 \mathrm{GHz}$ and $-13.8 \mathrm{~dB}$, with a gap of $30 \mu \mathrm{m}$ between electrodes. This was compared with the HFSS simulation with the same gap

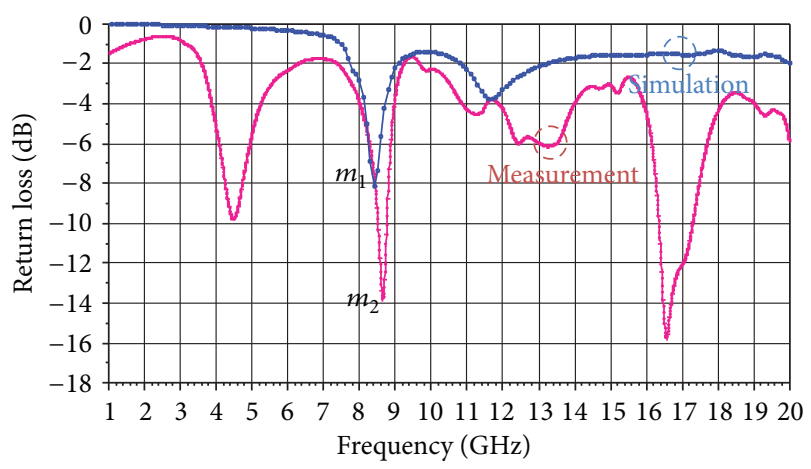

FIGURE 12: Simulated and measured return loss versus frequency for the MEMS-MTM antenna when the gap is $30 \mu \mathrm{m}$. The marker $m_{1}$ is at $8.4 \mathrm{GHz}$ and $-8.164 \mathrm{~dB}$, whereas $m_{2}$ is at $8.65 \mathrm{GHz}$ and $-13.803 \mathrm{~dB}$.

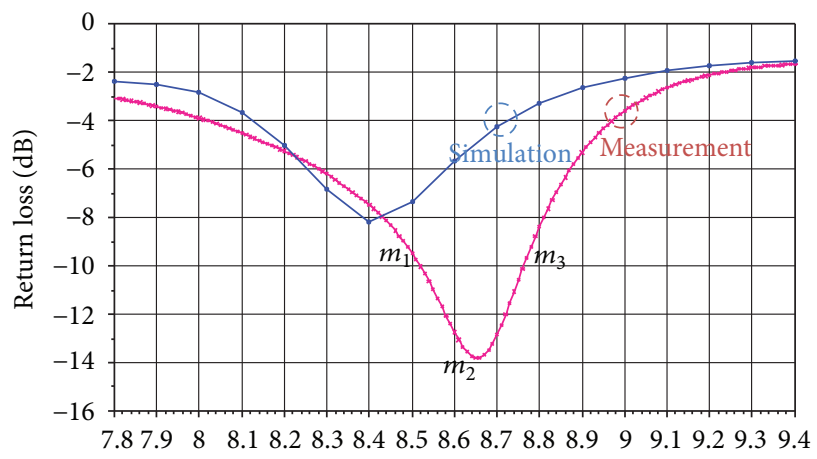

FIGURE 13: Measured and simulated return loss for the antenna. The markers $m_{1}$ is at $8.52 \mathrm{GHz}$ and $-10.027 \mathrm{~dB}, m_{2}$ is at $8.65 \mathrm{GHz}$, and $-13.803 \mathrm{~dB}$ and $m_{3}$ is at $8.76 \mathrm{GHz}$ and $-10.12 \mathrm{~dB}$.

between electrodes, which indicated a resonant frequency of $8.4 \mathrm{GHz}$ and $-8.16 \mathrm{~dB}$.

A comparison of the measured and simulated return loss $\left(\mathrm{S}_{11}\right)$ of the antenna and feed line is shown in Figure 13. The slight difference between the measured and simulated values can be attributed to fabrication imperfections and variations of material properties, such as silicon dielectric constant, and metal loss. The return loss for the antenna is better than $10 \mathrm{~dB}$ across the $8.52-8.76 \mathrm{GHz}$ frequency ranges, which translates to a $10 \mathrm{~dB}$ bandwidth of $3 \%$. The VSWR obtained from the measured $S_{11}$ is better than 1.5 throughout this frequency range. In addition, the MEMS-MTM antenna presented in this work has a $26 \mathrm{x}$ smaller footprint than similar antennas fabricated with microstrip technology.

The Quality-Factor (Q-Factor) for the antenna is defined as the ratio of the stored and radiated energies. In this case it is obtained by considering to the antenna as a resonant circuit using the $\mathrm{Y}$ admittance matrix. In this case, the Q-Factor $=$ absolute (imaginary $\left.\left(\mathrm{Y}\left(\mathrm{S}_{11}\right)\right) / \operatorname{real}\left(\mathrm{Y}\left(\mathrm{S}_{11}\right)\right)\right)$ is illustrated in Figure 14.

The efficiency and gain of antennas fabricated on thin silicon wafers are in general low; however, they are expected to be higher with the proposed design. This can be achieved 


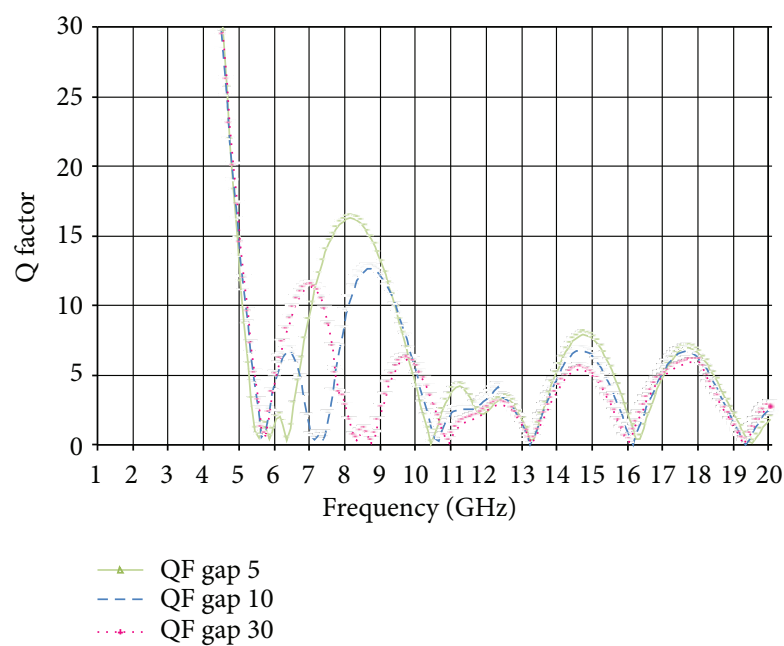

FIGURE 14: Q factor of the antenna when the gap between electrodes is 5,10 , and $30 \mu \mathrm{m}$.

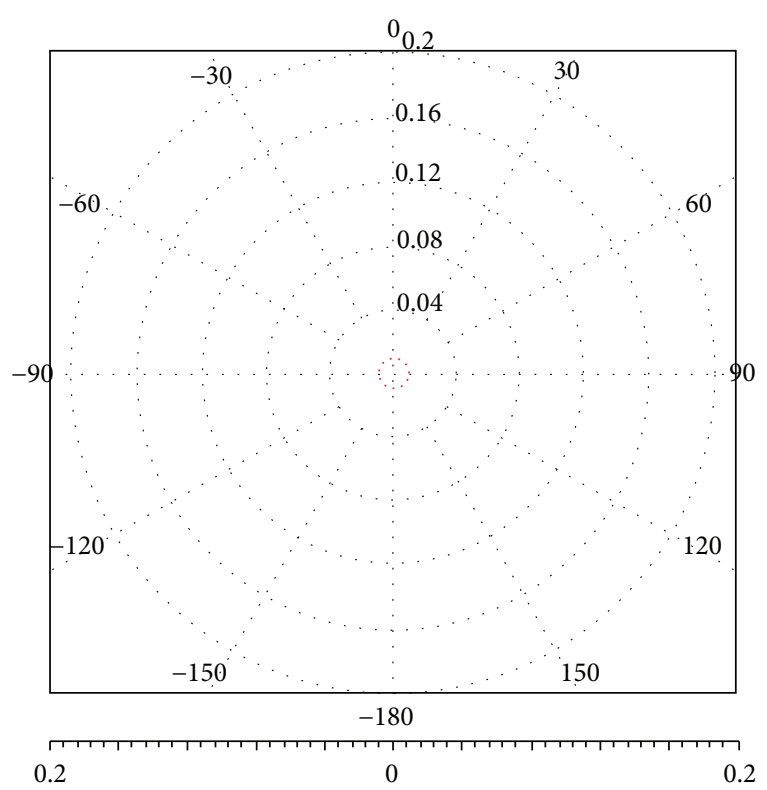

FIGURE 15: Antenna efficiency when the gap between electrodes is $30 \mu \mathrm{m}$.

by reducing substrate losses and surface waves using a highresistivity silicon substrate. Therefore, a tradeoff has to be made between substrate height and cell number, ease of fabrication and efficiency/gain.

Figure 15 shows the efficiency of the antenna when the gap is $30 \mu \mathrm{m}$.

\section{Conclusions}

In this paper, the design, fabrication, and measurements of an MEMS-MTM antenna as a basic cell have been described. This device incorporates metamaterial properties, which allows for the reduction in size compared to others for the same frequency range, and the inclusion of variable MEMS capacitors allow for configurability of the response. This combination has allowed a reduction in size of better than 26X since the response is determined by the constitutive $L_{L}$ and $C_{L}$ parameters rather than the wavelength.

Furthermore, the fabrication process as presented here, which incorporates a novel release procedure, is totally compatible with most integrated circuit fabrication processes, permitting the incorporation of additional circuitry in the same chip. The measurements performed on this device, once compared with simulations, validate the design, which is now being improved considering the residual stress gradient effect, which allows for a wide movement between electrodes and therefore tuning in frequency. The upper plates of these capacitors function as the radiating element, thus further reducing the area needed.

Furthermore, the bandwidth and efficiency of the antenna can be improved by increasing the number of cells, which makes it capable of meeting the requirements for many applications using RF wireless circuits.

\section{Conflict of Interest}

There is no conflict of interest with CoventoWare since both the INAOE and USF have valid licenses to use this program.

\section{Acknowledgments}

The authors wish to acknowledge CONACyT, Mexico, for the partial support of this work through Grant 83774-Y, and FORDECyT project no. 115976. G. Rosas Guevara also thanks CONACyT for the scholarship to undertake doctoral studies, no. 102735, and for the support in carrying out this research. Special recognition is due to the Nanotechnology Research and Education Center (NREC) at USF for their valuable support during the fabrication of the devices.

\section{References}

[1] C. Caloz and T. Itoh, "Application of the transmission line theory of left-handed (LH) materials to the realization of a microstrip LH transmission line," in Proceedings of the IEEEAP-S USNC/URSI National Radio Science Meeting, vol. 2, pp. 412-415, San Antonio, Tex, USA, 2002.

[2] C. Caloz and T. Itoh, "Novel microwave devices and structures based on the transmission line approach of meta-materials," in Proceedings of the IEEE MTT-S International Symposium, vol. 1, pp. 195-198, Philadelphia, Pa, USA, June 2003.

[3] C. Caloz and T. Itoh, Electromagnetic Metamaterials: Transmission Line Theory and Microwave Applications, John Wiley \& Sons, 2006.

[4] W. Tong, Z. Hu, H. S. Chua, P. D. Curtis, A. A. P. Gibson, and M. Missous, "Left-handed metamaterial coplanar waveguide components and circuits in GaAs MMIC technology," IEEE Transactions on Microwave Theory and Techniques, vol. 55, no. 8, pp. 1794-1800, 2007.

[5] V. G. Veselago, "The electrodynamics of substances with simultaneously negative values of $\varepsilon$ and $\mu$," Soviet Physics Uspekhi, vol. 10, no. 4, pp. 509-514, 1968. 
[6] Agilent ADS, http://www.home.agilent.com/agilent/product.js px?nid=-34360.0.00\&cc=MX\&lc=spa.

[7] Ansoft HFSS, http://www.ansoft.com.

[8] Coventorware, http://www.coventor.com.

[9] S. Pamidighantam, R. Puers, K. Baert, and H. A. C. Tilmans, "Pull-in voltage analysis of electrostatically actuated beam structures with fixed-fixed and fixed-free end conditions," Journal of Micromechanics and Microengineering, vol. 12, no. 4, pp. 458-464, 2002.

[10] D. A. Czaplewski, C. W. Dyck, H. Sumali et al., "A soft-landing waveform for actuation of a single-pole single-throw ohmic RF MEMS switch," Journal of Microelectromechanical Systems, vol. 15, no. 6, pp. 1586-1594, 2006.

[11] G. Rosas, Reconfigurable Microwave Circuits [Doctoral Dissertation], INAOE, 2011. 

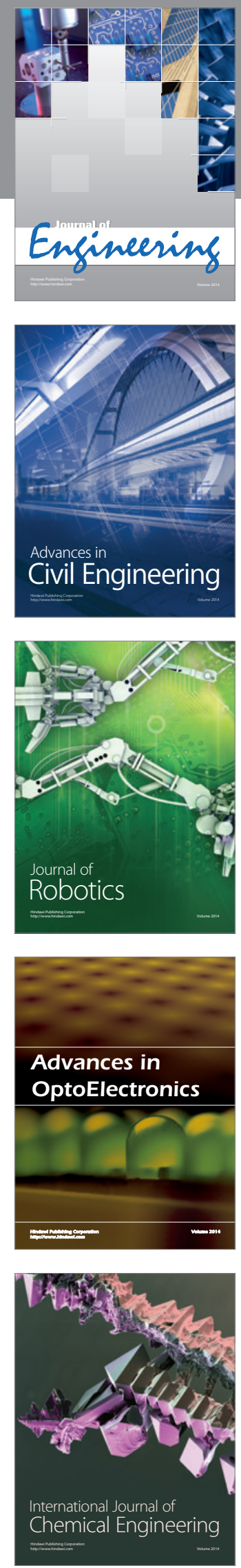

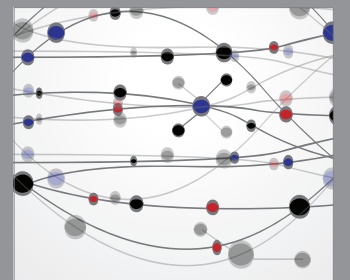

The Scientific World Journal
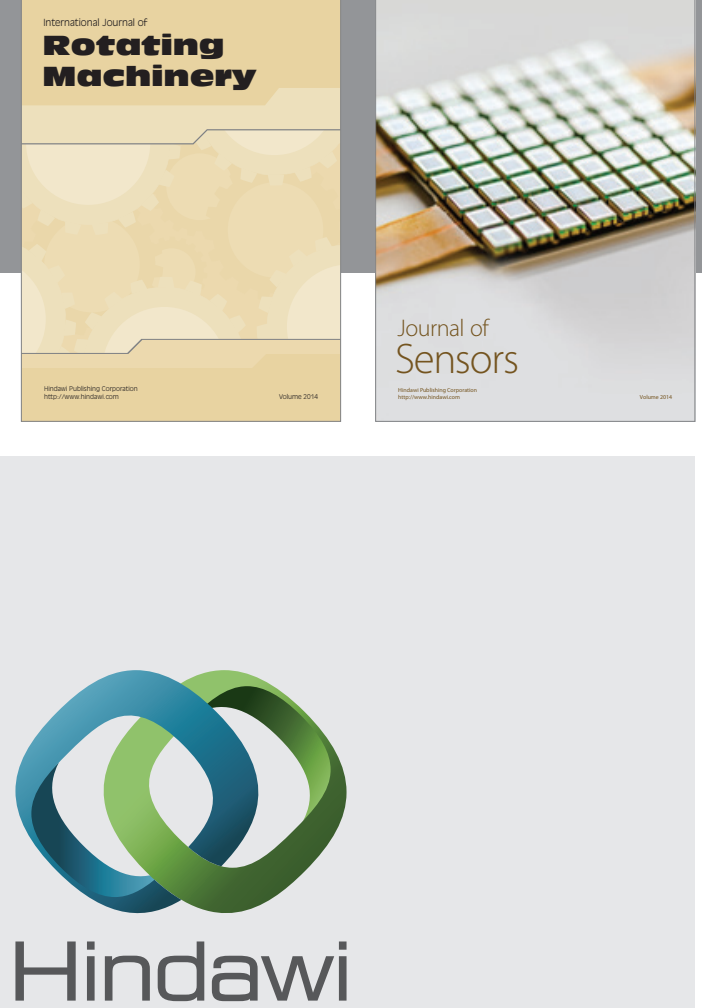

Submit your manuscripts at http://www.hindawi.com
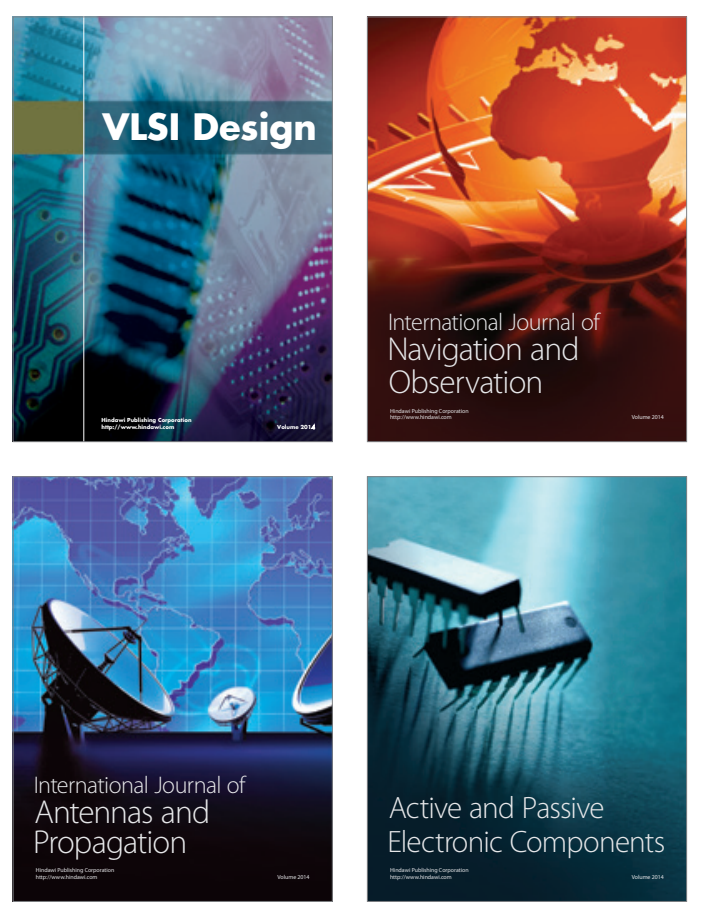
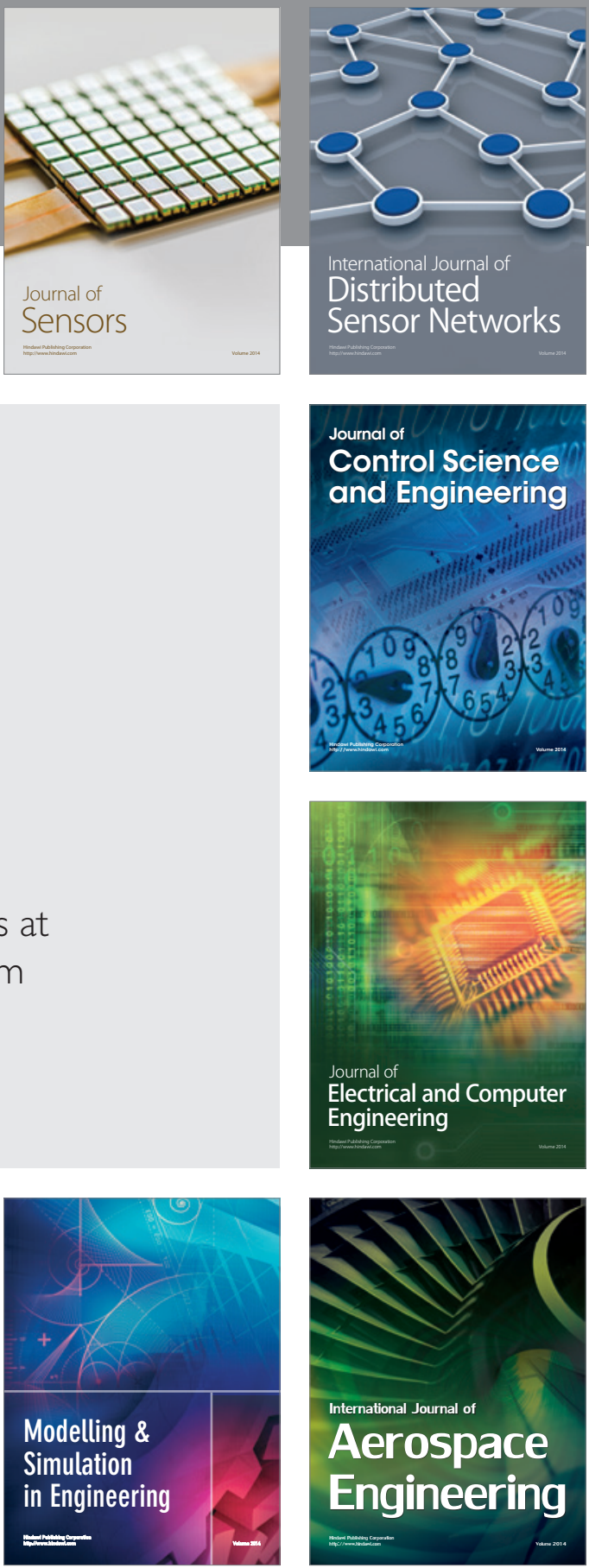

Journal of

Control Science

and Engineering
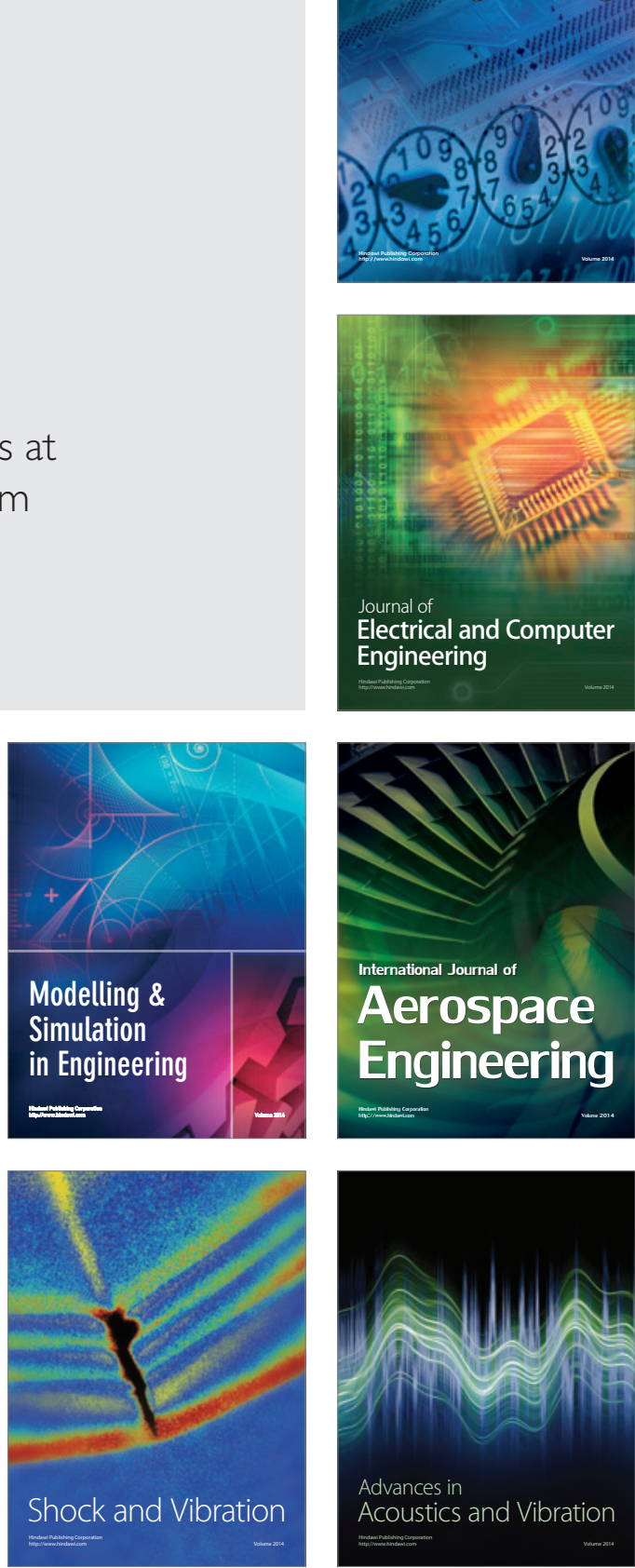